\title{
Unidirectional magneto-transport of linearly dispersing topological edge states
}

\author{
Zhanning Wang, ${ }^{1,2}$ Pankaj Bhalla, ${ }^{2,3}$ Mark Edmonds, ${ }^{4}$ Michael S. Fuhrer, ${ }^{4}$ and Dimitrie Culcer ${ }^{1,2}$ \\ ${ }^{1}$ School of Physics, The University of New South Wales, Sydney 2052, Australia \\ ${ }^{2} A R C$ Centre of Excellence in Future Low-Energy Electronics Technologies, \\ The University of New South Wales, Sydney 2052, Australia \\ ${ }^{3}$ Beijing Computational Science Research Center, Beijing 100193, China \\ ${ }^{4}$ ARC Centre of Excellence in Future Low-Energy Electronics Technologies, Monash University, Clayton, Victoria 3800
}

(Dated: August 13, 2021)

\begin{abstract}
Quantum spin-Hall edges are envisaged as next-generation transistors, yet they exhibit dissipationless transport only over short distances. Here we show that in a diffusive sample, where charge puddles with odd spin cause back-scattering, a magnetic field drastically increases the mean free path and drives the system into the ballistic regime with a Landauer-Buttiker conductance. A strong non-linear non-reciprocal current emerges in the diffusive regime with opposite signs on each edge, and vanishes in the ballistic limit. We discuss its detection in state-of-the-art experiments.
\end{abstract}

Quantum spin-Hall insulators are a novel class of materials hosting gapless, topologically protected, counter-propagating edge states. These have opposite spin polarizations and exhibit strong spin-momentum locking due to the dominant role of the spin-orbit interaction [1-9]. Time-reversal symmetry ensures edge states come in Kramers doublets, which cannot be back-scattered by time-reversal invariant perturbations [10-17]. Materials possessing topological edge states include topological insulators such as $\mathrm{HgTe}$ and $\mathrm{Bi}_{2} \mathrm{Se}_{3}$, Weyl semimetals such as $\mathrm{WTe}_{2}$, and Dirac semimetals such as $\mathrm{Na}_{3} \mathrm{Bi}$ [3, 18-24].

A ballistic edge has a longitudinal conductance of $e^{2} / h$ at low temperature, a fact that has led to proposals for using topological edge states as building blocks for next-generation transistors, exploiting electrically tunable topological phase transitions [25]. Nevertheless, following the experimental discovery of topological edge states, it has emerged that puddles with odd numbers of charges, which exist inherently in the host materials due to doping disorder in fabrication, can act as effective magnetic impurities that back-scatter the edge states and significantly reduce their mobility $[12,13,26-29]$. This may explain why ballistic conductance has only been observed over spatial scales of the order of $50 \mathrm{~nm}$ [19$21,23,26,30-32]$. Whereas initial studies focused on the Kondo effect, the Kondo temperature in current samples is expected to be negligibly small [33-35], while other aspects of transport remain poorly understood $[2,3,17$, 36-42]. The unexpectedly large resistance of topological edge states has emerged as a fundamental question and an obstacle in the development of topological transistors $[43,44]$. Bearing in mind the role of magnetic impurities, the first step in overcoming this problem is understanding edge magneto-transport in the presence of puddles. This includes the identification of non-reciprocal currents, since non-linear response probes interactions that are difficult to access in linear response, due to constraints imposed by mirror symmetry and Onsager relations [19, 45-48]. The rich physics underlying non-linear

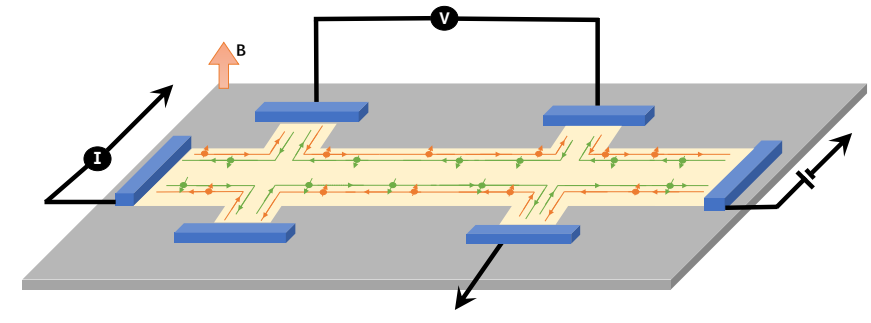

FIG. 1. Experimental setup in a perpendicular magnetic field B. The current is measured across the sample, while the voltage can be measured at two different terminals along one side. On the opposite side one terminal is grounded. Spin-up electrons are shown in orange, spin-down electrons in green.

phenomena [22, 49-53] has been manifest in recent discoveries such as Hall effects in time-reversal invariant systems, as well as in unexpected features of topological edges, such as a large uni-directional magneto-resistance at zero magnetic field [19, 20, 22, 48].

In this article we demonstrate that a magnetic field has a drastic effect on both the linear and non-linear response of topological edge states: (i) It enhances the mean free path $l$ by orders of magnitude without opening a gap, eventually driving the system into the ballistic regime; (ii) By breaking mirror symmetry the magnetic field enables a strong unidirectional non-linear electrical response in the diffusive regime. The direction of the current is determined by the magnetic field and the spin quantization axis, and it has a different sign on each edge. Interestingly, the non-reciprocal current vanishes in the ballistic regime. This reflects the fact that, once magnetic impurity scattering is surmounted, the only remaining magnetic interaction is the Zeeman interaction with the out of plane field, which can be gauged away. Whereas a complete description of charge puddles is beyond the scope of this work, modelling the puddles as magnetic impurities is a simple way of capturing the physics that governs their magnetoresistance, which is in excellent agreement with experiment [31]. 
(a)

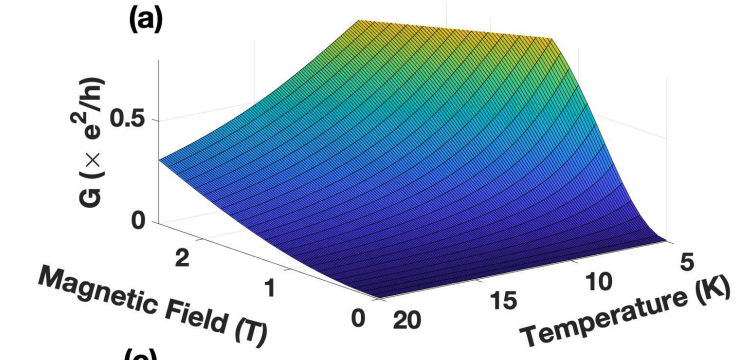

(c)

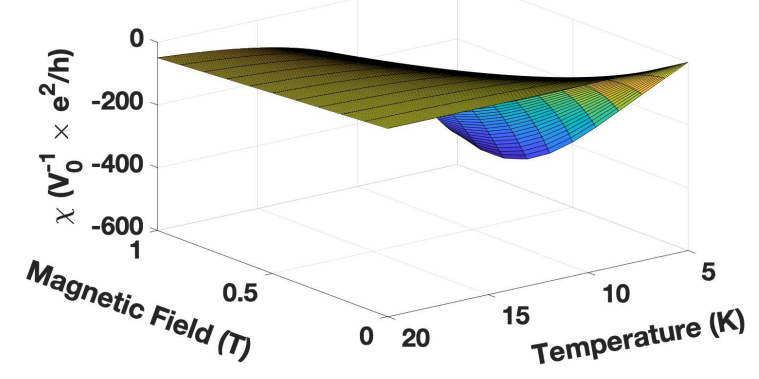

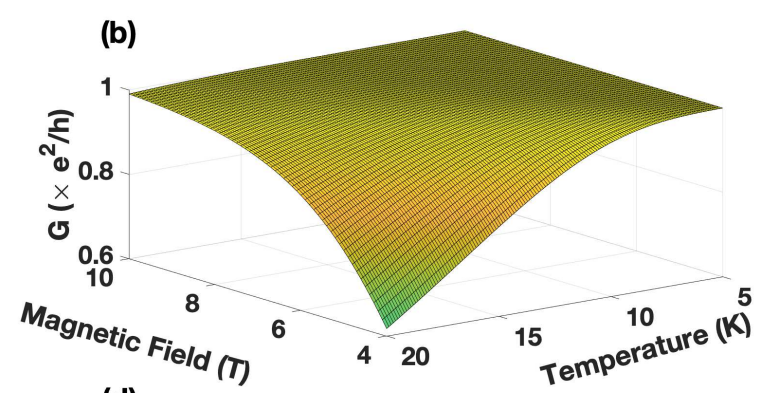

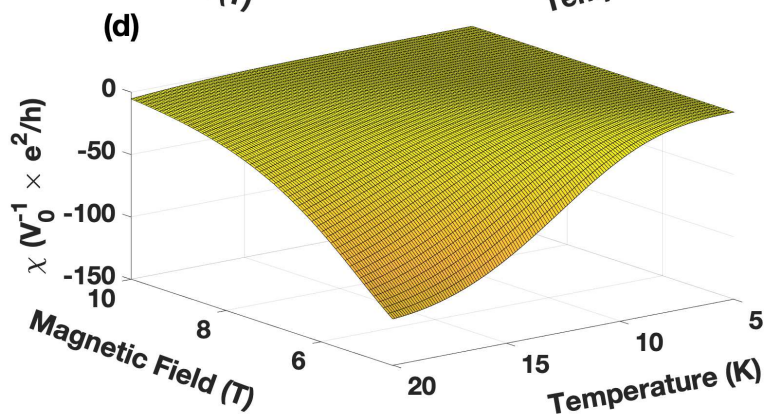

FIG. 2. Linear and non-linear response for a sample with $d=1000 \mathrm{~nm} ; v_{0}=10^{3} \mathrm{~V}$ is a scaling factor. (a) The conductance in the diffusive regime. When the magnetic field is small, the conductance increases as back-scattering is suppressed and the mean free path increases. (b) The conductance in the ballistic limit, when the mean free path is larger than the size of the sample, the conductance will not change, converging to to $e^{2} / h$. (c) The nonlinear response function $\chi$ in the diffusive regime. When the magnetic field is small, the mean free path is much shorter than the size of the sample, and the non-linear response increases as a function of B. (d) The nonlinear response function $\chi$ in the ballistic regime. At large magnetic fields the mean free path exceeds the size of the sample, causing $\chi$ to decrease in the ballistic regime and eventually vanish.

Referring to the set-up shown in Fig. 1, our main results are summarised in Fig. 2. The current in the channel will be denoted by $I$ and the potentials of the left and right electrodes by $V_{L}, V_{R}$ respectively. We define the conductance $G$ and the non-linear electrical response function $\chi$ by $I=G\left(V_{L}-V_{R}\right)+\chi\left(V_{L}-V_{R}\right)^{2}$. In Fig. 2-(a) and Fig. 2-(b) we have plotted the conductance $G$ as a function of the applied out-of-plane magnetic field $B$ at small and large values of $B$, where small and large are quantified below. It is seen that $G$ increases with $B$ and eventually reaches the quantized Landauer-Buttiker value of $e^{2} / h$, indicating that the system reaches the ballistic limit. This opens up the exciting possibility of using a ferromagnet with an out-of-plane magnetization as a practical method to increase the mean free path and to study transport in the ballistic regime. The ferromagnet could couple to the impurities either via a magnetic field or through the exchange interaction. Next, Fig. 2-(c) and Fig. 2-(d) show the non-linear electrical response function $\chi$ at small and large magnetic fields respectively. At small $B, \chi$ increases with $B$, but in contrast to the Ohmic term the non-linear signal reaches a maximum beyond which it decreases, tending to zero as the system reaches the ballistic regime. This vanishing response is a characteristic of the Dirac cone, indicating that the non-linear response is a probe of the edge state dispersion, and is a unique experimental signature reflecting chiral conduction in the TI. To generate and detect the second-order response at low-frequency it is sufficient to use an oscillator with angular frequency $\omega$ and read off the signal at $2 \omega$.

We focus on $\mathrm{Na}_{3} \mathrm{Bi}$ as a prototype material, motivated by the observations that ultra-thin films of $\mathrm{Na}_{3} \mathrm{Bi}$ have a band-gap of $\geq 300 \mathrm{meV}$ [54], much greater than $k_{B} T$ at room temperature, are robust to layer-number fluctuations caused by imperfect growth [55], exhibit an electrically driven topological phase transition[56], and show clear evidence of edge transport over millimetre distances, as well as a giant negative magneto-resistance [31]. Our model also applies to topological insulators with inversion symmetry such as $\mathrm{Bi}_{2} \mathrm{Se}_{3}$. Materials without inversion symmetry, such as $\mathrm{WTe}_{2}$, exhibit a positive magneto-resistance and a position-dependent spin quantization axis, so they fall outside our scope.

Considering a sample of finite size $d$ a magnetic field $\boldsymbol{B} \| \hat{\boldsymbol{z}}$ is applied out of the plane. The full Hamiltonian $H$ can be written as $H=H_{0}+H_{Z}+V+U+U_{Z}$, where the band Hamiltonian $H_{0}=\hbar v_{F} k_{x} \sigma_{z}$ represents the edge state dispersion of $\mathrm{Na}_{3} \mathrm{Bi} ; H_{Z}=g_{0} \mu_{B} B \sigma_{z}$ is the Zeeman interaction with the magnetic field. Since in the absence of warping terms the magnetic field does not open a gap in the dispersion the topological character of the states is 


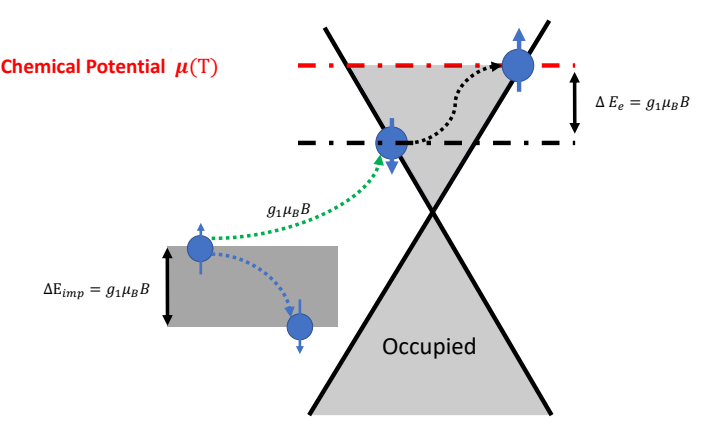

FIG. 3. Spin-flip scattering. An electron with spin down is scattered into a spin-up state, which, due to spin-momentum locking, travels in the opposite direction; the energy change is given by the impurity Zeeman splitting $E_{1, Z}=g_{1} \mu_{B} B$.

preserved (the role of warping is discussed briefly below). $V(x)$ is the electrostatic potential, with the associated electric field $E=-\partial V / \partial x$, and $-e$ the electron charge. The random magnetic impurity potential $U=$ $J s \cdot \sum_{i} S_{i} \delta\left(x-x_{i}^{\prime}\right)$ is the contact-like interaction term allowing spin-dependent scattering between an electron and effective magnetic impurities. $s$ indicates the spin operators of the electron and $S_{i}$ indicates the spin operators of impurities with spin- $1 / 2$ sited at position $x_{i}$. At the end we average over uncorrelated impurities which are all assumed to experience the same exchange interaction $J$ with the mobile carriers. We choose the impurity density and exchange coupling to reproduce the mean free path observed experimentally in the diffusive regime. The local moments are in thermal equilibrium, relaxing their energy and angular momentum rapidly to an external bath [57], which corresponds to what is seen experimentally. If the local moments coupled only to the edges the moments on each edge would polarize quickly and the edges would become ballistic in a short amount of time: this is not observed in experiment. The Zeeman interaction between the impurities and the out-of-plane magnetic field $U_{Z}=g_{1} \mu_{B} \sum_{i} S_{i} \cdot B$. The notation $\sigma_{z}$ in the full Hamiltonian represents the $z$-component of the Pauli spin matrix for a spin-1/2 particle. We focus on the DC limit, where $\omega \tau \ll 1$.

The negative magneto-resistance in Fig. 2 is explained by the relationship between the magnetic field and the mean free path. Although conventional 1D systems are either ballistic or localised, the notion of a mean free path, defined explicitly below, can be applied meaningfully to $1 \mathrm{D}$ topological edge states, a diffusive system in which localisation is nevertheless not expected due to topological protection. We note that up and down spins have the same mean free path $l$. Figure 3 gives a diagrammatic example of spin-flip scattering, showing a spin-down electron being flipped to the spin-up channel due to scattering off an impurity. The energy required

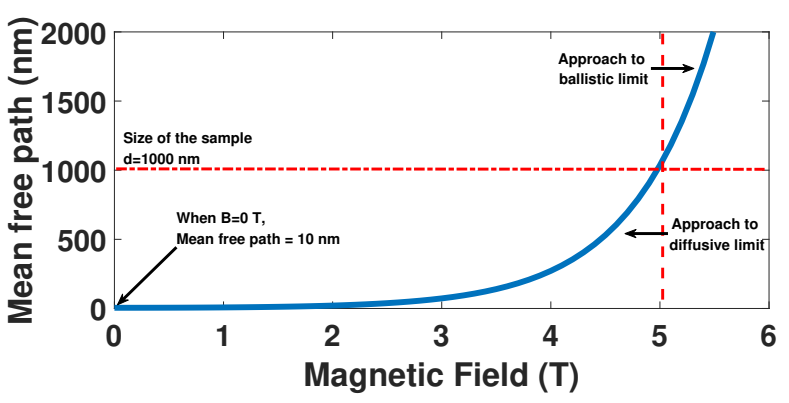

FIG. 4. The mean free path as a function of magnetic field. The red dashed lines mark the size of the sample $d=1000 \mathrm{~nm}$ compared with the mean free path the system. When the magnetic field is small, the system is diffusive, however a larger magnetic field will enhance the mean free path, driving the system into the ballistic regime, leading to a vanishing non-linear response. In all the plots, we have set the mean free path at zero magnetic field to be $10 \mathrm{~nm}$.

for this transition is set by the Zeeman splitting of the impurity spin states. As the magnetic field increases the energy cost likewise increases and the transition is suppressed. Figure 4 shows the mean free path $l$ increasing as a function of $\boldsymbol{B}$ until it exceeds the size $d$ of the sample. Based on this we define the diffusive regime as $l \ll d$, and the ballistic regime as $l>d$. We focus on these two limiting cases, in which simplifying approximations can be made. Specifically, in the diffusive regime one may assume a constant electric field across the channel, and the conductance takes the simple general form $G=\frac{e^{2}}{h} \frac{l}{d}$, where the entire magnetic field dependence is contained in the mean free path $l(B)$. In the ballistic regime it is straightforward to express the current as a function of the potential difference between the source and drain electrodes, and the potential drop occurs overwhelmingly in the vicinity of the electrodes due to contact resistance, although the exact potential profile is immaterial [58, 59]. The conductance is obtained straightforwardly as $G=\frac{e^{2}}{h}$. The intermediate region is complicated by potential fluctuations, and is not a focus of current experimental efforts. A full treatment requires accounting for screening thoroughly [60,61].

A perpendicular magnetic field breaks mirror symmetry and enables a second-order response, which increases as a function of the magnetic field in the diffusive regime due to the reduced efficiency of impurity scattering. Nevertheless, the non-linear response vanishes in the ballistic regime. Once transport becomes ballistic there is no more scattering and the impurities become irrelevant. The effective Hamiltonian becomes simply that of a Dirac cone, $H_{0}+H_{Z}$, whence $B$ can be removed by redefining the origin. The second-order response therefore probes the edge state dispersion: if a non-linear response is detected in the ballistic regime it must come from band structure terms of higher order 
in the wave vector, which are challenging to calculate computationally for $1 \mathrm{D}$ systems. Although they can be determined by symmetry their magnitude is generally unknown [30] (the details are reserved for a future publication).

The response of the other edge can be found by reflecting the Hamiltonian in the $x z$-plane. The Hamiltonian describing the dispersion for the other edge reads $H_{0}=-\hbar v_{F} k_{x} \sigma_{z}$. When the magnetic field is flipped the conductance $G$ does not change, consistent with Onsager symmetry. But the direction of the non-linear response on each edge is set by the spin orientation with respect to the magnetic field and the solution to the second order quantum kinetic equation changes sign for the other edge. Hence $\chi$ changes sign, ensuring time reversal breaking in the non-linear response function [49].

We derive a quantum kinetic equation following the procedure of Refs. 33 and 62, which ensures the Pauli blocking terms are correctly accounted for [62]. The full details are provided in the Supplemental material (SM) [63]. The system is described by the density matrix $\rho$, which satisfies the quantum Liouville equation $\partial \rho / \partial t+$ $(i / \hbar)[H, \rho]=0$. The explicit position dependence must be taken into account due to the finite size of the sample. Following a Wigner transformation [64, 65]

$$
\frac{\partial \rho}{\partial t}+\frac{1}{2 \hbar}\left\{\frac{\partial H_{0}}{\partial k_{x}}, \frac{\partial \rho}{\partial x}\right\}+J(\rho)=-\frac{e}{\hbar} \frac{\partial V}{\partial x} \frac{\partial \rho}{\partial k_{x}}
$$

In Eq. (1), the single particle density matrix $\rho$ takes the form $\operatorname{diag}\left\{f_{\uparrow}, f_{\downarrow}\right\}$. We write $f_{\uparrow}=f_{\uparrow}^{(0)}+g_{\uparrow}$ is the non-equilibrium distribution for the spin-up electrons composed of the equilibrium part $f_{\uparrow}^{(0)}$ and out-of-equilibrium part $g_{\uparrow}$; similarly, $f_{\downarrow}=f_{\downarrow}^{(0)}+g_{\downarrow}$ is the non-equilibrium distribution for the spin-down electrons, the equilibrium distribution have the form $f^{(0)}(\varepsilon)=[1+\exp (\beta(\varepsilon-\mu))]^{-1}$ where $\beta=\left(k_{B} T\right)^{-1}$. The last term in Eq. (1) is the scattering term in the Born approximation, which take the form

$$
\begin{aligned}
& J\left(g_{\uparrow}\right)=\int\left[P_{k \downarrow, k^{\prime} \uparrow} f_{\downarrow}^{\prime}\left(1-f_{\uparrow}\right)-P_{k \uparrow, k^{\prime} \downarrow} f_{\uparrow}\left(1-f_{\downarrow}^{\prime}\right)\right] \frac{d k^{\prime}}{2 \pi} \\
& J\left(g_{\downarrow}\right)=\int\left[P_{k \uparrow, k^{\prime} \downarrow} f_{\uparrow}^{\prime}\left(1-f_{\downarrow}\right)-P_{k \downarrow, k^{\prime} \uparrow} f_{\downarrow}\left(1-f_{\uparrow}^{\prime}\right)\right] \frac{d k^{\prime}}{2 \pi}
\end{aligned}
$$

Here $P^{i}\left(k^{\prime}, \downarrow \rightarrow k, \uparrow\right)$ indicates the probability of spin-flip scattering between a spin-up electron at $k$ and an impurity, ending with a spin-down electron at $k^{\prime}$. Primed quantities indicate the final state following a scattering event. We obtain two coupled Boltzmann equations for the spin-up and spin-down electrons:

$$
\begin{gathered}
\frac{\partial g_{\uparrow}(\varepsilon)}{\partial x}+\Gamma_{1}(\varepsilon) g_{\uparrow}(\varepsilon)-\Gamma_{2}(\varepsilon) g_{\downarrow}\left(\varepsilon_{-}\right)=-e \frac{\partial V}{\partial x} \frac{\partial f_{\uparrow}^{(0)}(\varepsilon)}{\partial \varepsilon} \\
\frac{\partial g_{\downarrow}(\varepsilon)}{\partial x}-\Gamma_{1}(\varepsilon) g_{\uparrow}\left(\varepsilon_{+}\right)+\Gamma_{2}\left(\varepsilon_{+}\right) g_{\downarrow}(\varepsilon)=-e \frac{\partial V}{\partial x} \frac{\partial f_{\downarrow}^{(0)}(\varepsilon)}{\partial \varepsilon}
\end{gathered}
$$

where $\varepsilon_{-}=\varepsilon-\varepsilon_{Z}$ and $\varepsilon_{+}=\varepsilon+\varepsilon_{Z}$ The two scattering rates are defined as follows:

$$
\begin{aligned}
& \Gamma_{1}(\varepsilon)=\frac{N_{i} J^{2}}{\hbar^{2} v_{F}}\left[\frac{1}{1+e^{-\alpha}}\left[1-f_{\downarrow}^{(0)}(\varepsilon)\right]+\frac{1}{1+e^{\alpha}} f_{\downarrow}^{(0)}(\varepsilon)\right] \\
& \Gamma_{2}(\varepsilon)=\frac{N_{i} J^{2}}{\hbar^{2} v_{F}}\left[\frac{1}{1+e^{-\alpha}} f_{\uparrow}^{(0)}(\varepsilon)+\frac{1}{1+e^{\alpha}}\left[1-f_{\uparrow}^{(0)}(\varepsilon)\right]\right],
\end{aligned}
$$

where $N_{i}$ is the number of impurities, the dimensionless factor $\alpha=g_{1} \mu_{B} B /\left(k_{B} T\right)^{-1}$, and the change of the Zeeman energy during the spin-flipping interactions $\varepsilon_{Z}=$ $g_{1} \mu_{B} B$. We solve the coupled Eq. (4) and Eq. (5) by integrating separately over left and right movers, which also ensures the correct solution in the ballistic regime:

$$
\begin{aligned}
& g_{\uparrow}^{(1)}=-e \int_{0}^{x}\left[\frac{\Gamma_{2}}{\kappa}+\frac{\Gamma_{1}}{\kappa} \exp \left[\kappa\left(x-x^{\prime}\right)\right]\right] \frac{\partial V}{\partial x} \frac{\partial f_{\uparrow}^{(0)}}{\partial \varepsilon} d x^{\prime}+e \int_{d}^{x}\left[\frac{\Gamma_{2}}{\kappa}\left(\exp \left[\kappa\left(x-x^{\prime}\right)\right]-1\right)\right] \frac{\partial V}{\partial x} \frac{\partial f_{\downarrow}^{(0)}}{\partial \varepsilon} d x^{\prime} \\
& g_{\downarrow}^{(1)}=-e \int_{d}^{x}\left[\frac{\Gamma_{1}}{\kappa}+\frac{\Gamma_{2}}{\kappa} \exp \left[\kappa\left(x-x^{\prime}\right)\right]\right] \frac{\partial V}{\partial x} \frac{\partial f_{\downarrow}^{(0)}}{\partial \varepsilon} d x^{\prime}+e \int_{0}^{x}\left[\frac{\Gamma_{1}}{\kappa}\left(\exp \left[\kappa\left(x-x^{\prime}\right)\right]-1\right)\right] \frac{\partial V}{\partial x} \frac{\partial f_{\uparrow}^{(0)}}{\partial \varepsilon} d x^{\prime}
\end{aligned}
$$

where $\kappa=\Gamma_{1}+\Gamma_{2}$, and the mean free path $l=$ $\kappa^{-1}=\left(\Gamma_{1}+\Gamma_{2}\right)^{-1}$. The current density $j=-e \operatorname{Tr}(v \rho)$, where $v=(1 / \hbar)\left(\partial H_{0} / \partial k\right)$. For spin-up electrons the momentum integration is performed over $k>0$, and for spin-down electrons over $k<0$. For the second-order response the sign of the magnetic field in the scattering terms Eq. (6), (7) will change, yet $\Gamma_{1}$ and $\Gamma_{2}$ are symmetric in $\alpha$; the sign of the driving term will change similarly to the band dispersion. The mean free path is unchanged and the formal solution to the differential equation is analogous to Eqs. 8-9, with the replacements $\frac{\partial f^{(0)}}{\partial \varepsilon} \rightarrow \frac{\partial g^{(1)}}{\partial \varepsilon}$. These equations cannot be reduced to a simple closed form and are solved iteratively.

In this work we have not discussed dispersions beyond the linear case. In $\mathrm{Bi}_{2} \mathrm{Se}_{3}$ warping complicates the dispersion, and in the spin eigenstate basis $H_{0}=A k_{x} \sigma_{y}+$ $C \sigma_{z} k_{x}^{3}$. A magnetic field $\| \hat{\boldsymbol{y}}$ yields a term of the form $\sigma_{z} B_{y}$ due to warping, which opens a small gap in the 
edge spectrum. However, warping only accounts for up to $10 \%$ of the Fermi energy, thus the gap is expected to be small, and will not influence the dynamics in the vicinity of the Fermi energy discussed here. Since the addition of warping complicates significantly the description of the interaction with the impurities, a further derivation is beyond the scope of this paper.

In summary, we have shown that a magnetic field drastically enhances the conductivity of topological edge states and gives rise to an edge-dependent non-linear response which vanishes in the ballistic limit. The magnetic field, as well as proximity to a ferromagnet, can be used to drive the system into the ballistic regime, while the non-linear response probes the edge state dispersion. In the future the transport theory can be extended to the Kondo regime along the lines of 33 .

Acknowledgments. This research is supported by the Australian Research Council Centre of Excellence in Future Low-Energy Electronics Technologies (project CE170100039) and funded by the Australian Government. PB acknowledges the National Key Research and Development Program of China (grant No. 2017YFA0303400), China postdoctoral science foundation (grant no. 2019M650461) and NSFC grant no. U1930402 for financial support.

[1] Jing Wang and Shou-Cheng Zhang, "Topological states of condensed matter," Nature Materials 16, 1062-1067 (2017).

[2] Pengke Li and Ian Appelbaum, "Interpreting current-induced spin polarization in topological insulator surface states," Phys. Rev. B 93, 220404 (2016).

[3] Chao-Xing Liu, Xiao-Liang Qi, HaiJun Zhang, Xi Dai, Zhong Fang, and Shou-Cheng Zhang, "Model hamiltonian for topological insulators," Phys. Rev. B 82, 045122 (2010).

[4] M. Z. Hasan and C. L. Kane, "Colloquium: Topological insulators," Rev. Mod. Phys. 82, 3045-3067 (2010).

[5] Xiao-Liang Qi, Taylor L. Hughes, and Shou-Cheng Zhang, "Topological field theory of time-reversal invariant insulators," Phys. Rev. B 78, 195424 (2008).

[6] Xiao-Liang Qi and Shou-Cheng Zhang, "Topological insulators and superconductors," Rev. Mod. Phys. 83, 1057-1110 (2011).

[7] C. L. Kane and E. J. Mele, "Quantum spin hall effect in graphene," Phys. Rev. Lett. 95, 226801 (2005).

[8] Arjun Mani and Colin Benjamin, "Probing helicity and the topological origins of helicity via non-local hanbury-brown and twiss correlations," Scientific Reports 7, 6954 (2017).

[9] S. Zhang and A. Fert, "Conversion between spin and charge currents with topological insulators," Phys. Rev. B 94, 184423 (2016).

[10] Markus König, Hartmut Buhmann, Laurens W. Molenkamp, Taylor Hughes, Chao-Xing Liu, Xiao-Liang Qi, and Shou-Cheng Zhang, "The quantum spin hall effect: Theory and experiment," Journal of the
Physical Society of Japan 77, 031007 (2008).

[11] Dimitrie Culcer, Aydın Cem Keser, Yongqing Li, and Grigory Tkachov, "Transport in two-dimensional topological materials: recent developments in experiment and theory," 2D Materials 7, 022007 (2020).

[12] B. V. Pashinsky, M. Goldstein, and I. S. Burmistrov, "Finite frequency backscattering current noise at a helical edge," Phys. Rev. B 102, 125309 (2020).

[13] Vladislav D. Kurilovich, Pavel D. Kurilovich, Igor S. Burmistrov, and Moshe Goldstein, "Helical edge transport in the presence of a magnetic impurity: The role of local anisotropy," Phys. Rev. B 99, 085407 (2019).

[14] Congjun Wu, B. Andrei Bernevig, and Shou-Cheng Zhang, "Helical liquid and the edge of quantum spin hall systems," Phys. Rev. Lett. 96, 106401 (2006).

[15] Rui-Lin Chu, Wen-Yu Shan, Jie Lu, and Shun-Qing Shen, "Surface and edge states in topological semimetals," Phys. Rev. B 83, 075110 (2011).

[16] Wang-Kong Tse and A. H. MacDonald, "Magneto-optical and magnetoelectric effects of topological insulators in quantizing magnetic fields," Phys. Rev. B 82, 161104 (2010).

[17] Jacob Linder, Yukio Tanaka, Takehito Yokoyama, Asle Sudbø, and Naoto Nagaosa, "Unconventional superconductivity on a topological insulator," Phys. Rev. Lett. 104, 067001 (2010).

[18] Joseph Maciejko, Taylor L. Hughes, and Shou-Cheng Zhang, "The quantum spin hall effect," Annual Review of Condensed Matter Physics 2, 31-53 (2011).

[19] Yanmeng Shi, Joshua Kahn, Ben Niu, Zaiyao Fei, Bosong Sun, Xinghan Cai, Brian A. Francisco, Di Wu, Zhi-Xun Shen, Xiaodong Xu, David H. Cobden, and Yong-Tao Cui, "Imaging quantum spin hall edges in monolayer $\mathrm{WTe}_{2}, "$ Science Advances 5 (2019), 10.1126/sciadv.aat8799.

[20] Zaiyao Fei, Tauno Palomaki, Sanfeng Wu, Wenjin Zhao, Xinghan Cai, Bosong Sun, Paul Nguyen, Joseph Finney, Xiaodong $\mathrm{Xu}$, and David H. Cobden, "Edge conduction in monolayer $\mathrm{WTe}_{2}$," Nature Physics 13, 677-682 (2017).

[21] Shujie Tang, Chaofan Zhang, Dillon Wong, Zahra Pedramrazi, Hsin-Zon Tsai, Chunjing Jia, Brian Moritz, Martin Claassen, Hyejin Ryu, Salman Kahn, Juan Jiang, Hao Yan, Makoto Hashimoto, Donghui Lu, Robert G. Moore, Chan-Cuk Hwang, Choongyu Hwang, Zahid Hussain, Yulin Chen, Miguel M. Ugeda, Zhi Liu, Xiaoming Xie, Thomas P. Devereaux, Michael F. Crommie, Sung-Kwan Mo, and Zhi-Xun Shen, "Quantum spin hall state in monolayer $1 \mathrm{~T}$ '-WTe 2 ," Nature Physics 13, 683-687 (2017).

[22] Wenjin Zhao, Zaiyao Fei, Tiancheng Song, Han Kyou Choi, Tauno Palomaki, Bosong Sun, Paul Malinowski, Michael A. McGuire, Jiun-Haw Chu, Xiaodong $\mathrm{Xu}$, and David H. Cobden, "Magnetic proximity and nonreciprocal current switching in a monolayer $\mathrm{WTe}_{2}$ helical edge," Nature Materials 19, 503-507 (2020).

[23] Chang Liu, Golrokh Akhgar, James L. Collins, Jack Hellerstedt, Cheng Tan, Lan Wang, Shaffique Adam, Michael S. Fuhrer, and Mark T. Edmonds, "Quantum transport in air-stable $\mathrm{Na}_{3} \mathrm{Bi}$ thin films," ACS Applied Materials \& Interfaces 12, 35542-35546 (2020).

[24] B. Andrei Bernevig, Taylor L. Hughes, and Shou-Cheng Zhang, "Quantum spin hall effect and topological phase transition in HgTe quantum wells," Science 314, 1757-1761 (2006). 
[25] Hui Pan, Meimei Wu, Ying Liu, and Shengyuan A. Yang, "Electric control of topological phase transitions in dirac semimetal thin films," Scientific Reports 5, 14639 (2015).

[26] Yuval Vinkler-Aviv, Daniel May, and Frithjof B. Anders, "Analytical and numerical study of the out-of-equilibrium current through a helical edge coupled to a magnetic impurity," Phys. Rev. B 101, 165112 (2020).

[27] Luca Vannucci, Thomas Olsen, and Kristian S. Thygesen, "Conductance of quantum spin hall edge states from first principles: The critical role of magnetic impurities and inter-edge scattering," Phys. Rev. B 101, 155404 (2020).

[28] T. Knispel, W. Jolie, N. Borgwardt, J. Lux, Zhiwei Wang, Yoichi Ando, A. Rosch, T. Michely, and M. Grüninger, "Charge puddles in the bulk and on the surface of the topological insulator $\mathrm{BiSbTeSe}_{2}$ studied by scanning tunneling microscopy and optical spectroscopy," Phys. Rev. B 96, 195135 (2017).

[29] Jukka I. Väyrynen, Moshe Goldstein, Yuval Gefen, and Leonid I. Glazman, "Resistance of helical edges formed in a semiconductor heterostructure," Phys. Rev. B 90, 115309 (2014).

[30] Artem Kononov, Gulibusitan Abulizi, Kejian Qu, Jiaqiang Yan, David Mandrus, Kenji Watanabe, Takashi Taniguchi, and Christian Schönenberger, "One-dimensional edge transport in few-layer $\mathrm{WTe}_{2}$," Nano Letters 20, 4228-4233 (2020).

[31] Chang Liu, Dimitrie Culcer, Zhanning Wang, Mark T. Edmonds, and Michael S. Fuhrer, "Helical edge transport in millimeter-scale thin films of $\mathrm{Na} 3 \mathrm{Bi}$," Nano Letters 20, 6306-6312 (2020).

[32] Pietro Novelli, Fabio Taddei, Andre K. Geim, and Marco Polini, "Failure of conductance quantization in two-dimensional topological insulators due to nonmagnetic impurities," Phys. Rev. Lett. 122, 016601 (2019).

[33] Jie Wang and Dimitrie Culcer, "Suppression of the kondo resistivity minimum in topological insulators," Phys. Rev. B 88, 125140 (2013).

[34] Qin Liu, Chao-Xing Liu, Cenke Xu, Xiao-Liang Qi, and Shou-Cheng Zhang, "Magnetic impurities on the surface of a topological insulator," Phys. Rev. Lett. 102, 156603 (2009).

[35] Huan-Wen Wang, Bo Fu, and Shun-Qing Shen, "Intrinsic magnetoresistance in three-dimensional dirac materials with low carrier density," Phys. Rev. B 98, 081202 (2018).

[36] Jukka I. Väyrynen and Leonid I. Glazman, "Current noise from a magnetic moment in a helical edge," Phys. Rev. Lett. 118, 106802 (2017).

[37] Doru Sticlet and Jérôme Cayssol, "Dynamical response of dissipative helical edge states," Phys. Rev. B 90, 201303 (2014).

[38] H. Z. Shen, M. Qin, X. Q. Shao, and X. X. Yi, "General response formula and application to topological insulator in quantum open system," Phys. Rev. E 92, 052122 (2015).

[39] Hai-Zhou Lu and Shun-Qing Shen, "Finite-temperature conductivity and magnetoconductivity of topological insulators," Phys. Rev. Lett. 112, 146601 (2014).

[40] T Ozturk, R L Field, Y S Eo, S Wolgast, K Sun, and C Kurdak, "Influence of helical spin structure on the magnetoresistance of an ideal topological insulator,"
Journal of Physics Communications 1, 035005 (2017).

[41] S. X. Zhang, R. D. McDonald, A. Shekhter, Z. X. Bi, Y. Li, Q. X. Jia, and S. T. Picraux, "Magneto-resistance up to 60 tesla in topological insulator $\mathrm{Bi}_{2} \mathrm{Te}_{3}$ thin films," Applied Physics Letters 101, 202403 (2012).

[42] Hong Yao and Dung-Hai Lee, "Topological insulators and topological nonlinear $\sigma$ models," Phys. Rev. B 82, 245117 (2010).

[43] S. Wiedmann, A. Jost, B. Fauqué, J. van Dijk, M. J. Meijer, T. Khouri, S. Pezzini, S. Grauer, S. Schreyeck, C. Brüne, H. Buhmann, L. W. Molenkamp, and N. E. Hussey, "Anisotropic and strong negative magnetoresistance in the three-dimensional topological insulator bi $\mathrm{bse}_{3}$," Phys. Rev. B 94, 081302 (2016).

[44] Yoichi Tanaka, A. Furusaki, and K. A. Matveev, "Conductance of a helical edge liquid coupled to a magnetic impurity," Phys. Rev. Lett. 106, 236402 (2011).

[45] Takuro Kobayashi, Taiki Matsushita, Takeshi Mizushima, Atsushi Tsuruta, and Satoshi Fujimoto, "Negative thermal magnetoresistivity as a signature of a chiral anomaly in weyl superconductors," Phys. Rev. Lett. 121, 207002 (2018).

[46] Shuichi Murakami, "Quantum spin hall effect and enhanced magnetic response by spin-orbit coupling," Phys. Rev. Lett. 97, 236805 (2006).

[47] B. A. Levitan and T. Pereg-Barnea, "Second-order topological insulator under strong magnetic field: Landau levels, zeeman effect, and magnetotransport," Phys. Rev. Research 2, 033327 (2020).

[48] Ebrahim Sajadi, Tauno Palomaki, Zaiyao Fei, Wenjin Zhao, Philip Bement, Christian Olsen, Silvia Luescher, Xiaodong Xu, Joshua A. Folk, and David H. Cobden, "Gate-induced superconductivity in a monolayer topological insulator," Science 362, 922-925 (2018).

[49] Yoshinori Tokura and Naoto Nagaosa, "Nonreciprocal responses from non-centrosymmetric quantum materials," Nature Communications 9, 3740 (2018).

[50] Kaifei Kang, Tingxin Li, Egon Sohn, Jie Shan, and Kin Fai Mak, "Nonlinear anomalous hall effect in few-layer $\mathrm{WTe}_{2}$," Nature Materials 18, 324-328 (2019).

[51] Su-Yang Xu, Qiong Ma, Huitao Shen, Valla Fatemi, Sanfeng Wu, Tay-Rong Chang, Guoqing Chang, Andrés M. Mier Valdivia, Ching-Kit Chan, Quinn D. Gibson, Jiadong Zhou, Zheng Liu, Kenji Watanabe, Takashi Taniguchi, Hsin Lin, Robert J. Cava, Liang Fu, Nuh Gedik, and Pablo Jarillo-Herrero, "Electrically switchable berry curvature dipole in the monolayer topological insulator $\mathrm{WTe}_{2}$," Nature Physics 14, 900-906 (2018).

[52] Pankaj Bhalla, Allan H. MacDonald, and Dimitrie Culcer, "Resonant photovoltaic effect in doped magnetic semiconductors," Phys. Rev. Lett. 124, 087402 (2020).

[53] Inti Sodemann and Liang Fu, "Quantum nonlinear hall effect induced by berry curvature dipole in time-reversal invariant materials," Phys. Rev. Lett. 115, 216806 (2015).

[54] I. Di Bernardo, J. Collins, W. Wu, Ju Zhou, Shengyuan A. Yang, Sheng Ju, M. T. Edmonds, and M. S. Fuhrer, "Importance of interactions for the band structure of the topological dirac semimetal $\mathrm{Na}_{3} \mathrm{Bi}$," Phys. Rev. B 102, 045124 (2020).

[55] Iolanda Di Bernardo, Jack Hellerstedt, Chang Liu, Golrokh Akhgar, Weikang Wu, Shengyuan A. Yang, Dimitrie Culcer, Sung-Kwan Mo, Shaffique Adam, 
Mark T. Edmonds, and Michael S. Fuhrer, "Progress in epitaxial thin-film $\mathrm{Na}_{3} \mathrm{Bi}$ as a topological electronic material," Advanced Materials 33, 2005897 (2019).

[56] James L. Collins, Anton Tadich, Weikang Wu, Lidia C. Gomes, Joao N. B. Rodrigues, Chang Liu, Jack Hellerstedt, Hyejin Ryu, Shujie Tang, Sung-Kwan Mo, Shaffique Adam, Shengyuan A. Yang, Michael S. Fuhrer, and Mark T. Edmonds, "Electric-field-tuned topological phase transition in ultrathin $\mathrm{Na}_{3} \mathrm{Bi}$," Nature 564, 390-394 (2018).

[57] Anders Mathias Lunde and Gloria Platero, "Helical edge states coupled to a spin bath: Current-induced magnetization," Phys. Rev. B 86, 035112 (2012).

[58] Supriyo Datta, Quantum Transport: Atom to Transistor (Cambridge University Press, 2005).

[59] Supriyo Datta, Electronic Transport in Mesoscopic Systems, Cambridge Studies in Semiconductor Physics and Microelectronic Engineering (Cambridge University Press, 1995).

[60] S. Adam, E. H. Hwang, and S. Das Sarma, "Two-dimensional transport and screening in topological insulator surface states," Phys. Rev. B 85, 235413 (2012).
[61] S. Das Sarma, E. H. Hwang, and Hongki Min, "Carrier screening, transport, and relaxation in three-dimensional dirac semimetals," Phys. Rev. B 91, 035201 (2015).

[62] F. T. Vasko and V. V. Mitin, "Generation and recombination processes via acoustic phonons in disordered graphene," Phys. Rev. B 84, 155445 (2011).

[63] The Supplemental material discusses, i) the full form of the quantum kinetic equation, ii) proof for the vanishing elastic scattering, iii) matrix element corresponds to the interaction with impurities, iv) calculation for the other edge, and v) scattering term with the addition of the tilt in the Hamiltonian.

[64] Dimitrie Culcer, Akihiko Sekine, and Allan H. MacDonald, "Interband coherence response to electric fields in crystals: Berry-phase contributions and disorder effects," Phys. Rev. B 96, 035106 (2017).

[65] Dimitrie Culcer, M. E. Lucassen, R. A. Duine, and R. Winkler, "Current-induced spin torques in iii-v ferromagnetic semiconductors," Phys. Rev. B 79, 155208 (2009). 